\title{
Corrigendum to "Squeezing flow of second grade liquid subject to non-Fourier heat flux and heat generation/absorption" [Colloid Polym. Sci. 295 (2017) 967-975]
}

\author{
T. Hayat $^{1,2} \cdot$ M. Waleed Ahmed Khan ${ }^{1} \cdot$ A. Alsaedi ${ }^{2} \cdot$ M. Ijaz Khan ${ }^{1}$
}

Received: 24 June 2017 / Revised: 22 September 2017 / Accepted: 29 September 2017 / Published online: 13 October 2017

(C) Springer-Verlag GmbH Germany 2017

Keywords Second grade fluid · Heat generation/absorption · Cattaneo-Christov heat flux $\cdot$ Squeezing flow $\cdot$ Variable thermal conductivity

In this recently published paper, we noted few typing errors. In this recently published paper, we noted a few typing errors. These may be read as follows:

$Q$ denotes the heat generation/absorption parameter when $Q>0 / Q<0$.

Here, $T_{h}$ and $T_{w}$ are the temperatures of the upper and the lower walls.

In Eq. (10), it should read no $\tau_{0}$ in the first term and no $\left(T-T_{h}\right)$ in the second term on RHS.

The values of heat generation/absorption $(\delta)$ and modified heat generation/absorption $\left(\delta_{1}\right)$ parameters in Eq. (14) are

$$
\begin{aligned}
& \delta=\frac{Q(1-c t)}{\rho c_{p} a}, \\
& \delta_{1}=\frac{\tau_{0} Q}{\rho c_{p}}=\gamma \delta, \\
& \gamma=\frac{\tau_{0} a}{(1-c t)}
\end{aligned}
$$

These parameters $(\delta)$ and $\left(\delta_{1}\right)$ should be given different values.

There should be no $\delta, \varepsilon$ and $\delta_{1}$ in the captions for Figs. 2a, 3 and 5.

1. Deborah number in conclusion (3) is second grade parameter.

M. Ijaz Khan

mikhan@math.qau.edu.pk

1 Department of Mathematics, Quaid-i-Azam University, Islamabad 44000, Pakistan

2 Nonlinear Analysis and Applied Mathematics (NAAM) Research Group, Department of Mathematics, Faculty of Science, King Abdulaziz University, P. O. Box 80257, Jeddah 21589, Saudi Arabia 